\title{
Better Sleep Experience for the Critically Ill: A Comprehensive Strategy for Designing Hospital Soundscapes
}

\author{
Dilip Birdja ${ }^{1, *}$ and Elif Özcan ${ }^{1,2, *}$ \\ 1 Critical Alarms Lab, Delft University of Technology, 2628 CE Delft, The Netherlands \\ 2 Department of Intensive Care, Erasmus Medical Center Rotterdam, 3015 CD Rotterdam, The Netherlands \\ * Correspondence: D.V.Birdja-1@tudelft.nl (D.B.); E.Ozcan@tudelft.nl (E.Ö.)
}

Received: 13 March 2019; Accepted: 15 May 2019; Published: 22 May 2019

\begin{abstract}
In this paper, the sleep phenomenon is considered in relation to critical care soundscapes with the intention to inform hospital management, medical device producers and policy makers regarding the complexity of the issue and possible modes of design interventions. We propose a comprehensive strategy based on soundscape design approach that facilitates a systematic way of tackling the auditory quality of critical care settings in favor of better patient sleep experience. Future research directions are presented to tackle the knowledge deficits in designing for critical care soundscapes that cater for patient sleep. The need for scientifically-informed design interventions for improving patient sleep experience in critical care is highlighted. The value of the soundscape design approach for resolving other sound-induced problems in critical care and how the approach allows for patient-centred innovation that is beyond the immediate sound issue are further discussed.
\end{abstract}

Keywords: critical care; patient sleep; hospital sounds; medical soundscapes; design strategy

\section{Introduction}

Sleep plays an important role in maintaining good health and therefore is an essential component in the well-being and quality of life of individuals. Maintaining the right quantity and quality of sleep should therefore be encouraged in short-term and long-term care settings [1]. The restorative property of sleep makes sleep an indispensable element in the recovery of patients and an important subject for clinicians to take care of. However, critical care settings with excessive number of alarms, loud interaction sounds, machinery noise and people conversing pose a threat to one's sleep experience either making falling asleep or maintaining good (i.e., uninterrupted) sleep difficult to achieve. The combination of being exposed to an unfamiliar auditory environment and lack of sleep are detrimental for the wellbeing of the already vulnerable critically ill patients. Such vulnerable patients rely heavily on good sleep, more than healthy individuals, as they are in the recovery or stabilization process. Earlier studies indicating the negative effect of sound in critical care settings also mention sound-induced sleep problems [2-4] and discuss the importance of new protocols and new design opportunities around sound in intensive care units [5]. The function of sleep in care settings as well as the physical properties of care environments (e.g., sound producing events) need to be further studied, if the aim is to avoid long-term problems induced by lack of sleep [6].

The main function of critical care environments entails patient health and its criticality. Many technological innovations and medical systems are designed to monitor patients and support their failing organs, prioritizing patient care over patient comfort. However, the definition of care can also extend to reducing the unwanted effects of the technology employed in critical care. Care activities in critical care are becoming a main threat for both patients and clinical staff causing anxiety, fatigue and 
other psychological and neurological disorder's such as delirium and post intensive care syndrome $[7,8]$ and high noise levels contribute to such unwanted affects [9].

Sound-induced sleep problems in critical care are also of great importance to hospital management, medical device producers and policy makers who constantly strive for improved patient care and safety [7]. Currently, these stakeholders adopt strategies to tackle the sleep problem from their own perspective, which results in individual efforts that are asynchronous, and solutions that might be incompatible with other stakeholder's concerns. Moreover, individual strategies are developed and applied locally not allowing for a standardized procedure to address the sound problem in relation to patient sleep. Furthermore, while these individual strategies might be effective in fighting the symptoms of a specific sound-originated problem, they fail to address it in a broader perspective. That is, critical care, by nature, induces an acoustically hostile environment due to many sound producing events taking place simultaneously. Therefore, it is necessary to unravel the complexity of the acoustic environment and embrace the notion that individual sound producing events should be regarded holistically in the broader context of soundscapes. Soundscapes are the combination of all acoustic resources, natural and artificial, within a given area as modified by the environment [10] and how they influence patient sleep in hospital environments is yet to be discovered.

In this paper, our aim is to develop a comprehensive strategy to improve patient sleep through reorganization and harmonization of critical care soundscapes. Our strategy stems from systematically studying the critical care context in terms of sleep phenomenon, critical care environment and patient-nurse experience in relation to sound producing events. This strategy is meant to encourage the involved stakeholders (hospital management, manufacturers of medical devices, and policy makers) to adopt a holistic approach for improving the patient's sleep experience. In the following sections, we first establish what is known about patient sleep in critical care and auditory quality of critical care settings in general and introduce the critical care soundscape in specific. We conclude the paper with presenting the structure of our strategy with its intended use illustrated through design interventions that have the potential to improve patient's sleep experience. Overall, the proposed strategy is meant to open new research directions as well as innovative design solutions, as unravelling the complexity of the critical care soundscapes will reveal knowledge gaps that can initiate design solutions.

\section{Effect of Medical Sounds on the Critically Ill Patient Sleep}

\subsection{Sleep Quality of the Critically Ill Patients}

It is widely accepted that sleep has restorative properties [11-13]: Uninterrupted sleep helps restore physical, cognitive, and emotional functioning of the body (e.g., reducing sensory input, balancing hormonal activities, and reorganizing memory). For the restorative function to be effective, one needs to undergo all four stages of sleep (three non-rapid eye movement (REM) stages and one REM stage). The third stage, often referred to as the deep sleep stage, is considered as the most restorative stage of sleep; thus, entering deep sleep is the gold standard to achieve and maintain physical health and subjective well-being. A good night sleep is characterized by the ability to go through the different sleep stages. Sleep cycles (non-REM and REM stages) have a duration of around $90 \mathrm{~min}$, therefore going through the different sleep stages happens repeatedly while sleeping. Waking up during these sleep cycles is possible and is often caused by arousals (i.e., an arousal is a sudden change in brain activity). The nature of these arousals can differ and can be triggered internally or externally. Arousals do not immediately lead to awakening of the patient, as it could merely cause a shift out of deeper sleep to the lighter phases of sleep, from which it is easier to be woken up.

Going through repeated sleep cycles during the night is indicative of having a proper night's rest or obtaining good sleep quality [14]. However, for critically ill patients having, repeated sleep cycles when asleep in a critical care environment is hard to achieve. The combination of pain and a plethora of external stimuli (alarms, medical devices, lights and interaction with clinical staff) make the critical care setting a place where proper sleep quality for patients is hard to obtain. For this reason, sleep 
abnormalities (i.e., sleep fragmentation, sleep disruption or lack of sleep) are common in critically ill patients, and this results in problems during and after hospitalization [14]. Some of these complications such as drowsiness, fatigue, confusion, emotional instability, and sometimes delirium emerge during hospital stay, however these negative episodes can induce PICS (post intensive care syndrome) and especially sounds can trigger the onset of intensive stress and fear in situations after hospitalization (symptoms similar to post-traumatic stress syndrome). Factors that play a role in the development of these abnormalities can be patient related (internal) or ICU related (external) or both and cause sleep fragmentation, sleep disruption and delirium. Pre-existing sleep disorders, pain and anxiety are a few of the patient related factors that have an impact on the development of sleep deprivation in the ICU. In addition, there are ICU related factors such as excessive noise, time-inappropriate lighting and constant patient care activities that can have an influence on the development of sleeping disorders as well [15].

Sleep deprivation and sleep disruption are just a few sleep abnormalities critically ill patients can suffer from. Studies further exploring sleep disturbances in critically ill patients (by using polysomnography) have found that, compared to healthy adults, critically ill patients have prolonged sleep latency, sleep fragmentation, decreased sleep efficiency and an increased number of arousals in comparison to healthy adults. Moreover, in the sleep architecture of critically ill patients, there is a predominance of stage 2 sleep, where stage 3 sleep and REM sleep are either absent or severely decreased. There is no significant discrepancy in the total of sleep time hours between healthy adults and critically ill patients, however around half of the sleep time of critically ill patients takes place during daytime, where the emphasis shifts towards the lighter stages of sleep [16-19].

Sleep is often explained and explored from the perspective of sleep disorders (e.g., sleep fragmentation and sleep latency), sleep quality or sleep quantity. However, sleep health-as a comprehensive understanding of what sleep entails-seems to be an underestimated concept. Buysse (2014) defined sleep health as "a multidimensional pattern of sleep-wakefulness, adapted to individual, social, and environmental demands, that promotes physical and mental well-being, characterized by subjective satisfaction, appropriate timing, adequate duration, high efficiency, and sustained alertness during waking hours" [20] (p. 12). Taking this perspective into consideration, current sleep protocols in care settings fail to allow room for setting individual preferences, as the patient's sleep experience is-next to more objective factors-defined by subjective factors as well.

Changing the influence that external factors (e.g., noise, light and patient care activities) have on the sleep experience of patients is most feasible from the perspective of designers, given that internal factors (e.g., pain, anxiety, and medication) affecting the patient's sleep experience are more difficult to tackle as conditions differ amongst patients and it is often not clear to what extent internal factors such as pain affect the patients' sleep.

\subsection{Auditory Quality of Critical Care}

One of the external, critical care related, factors that has the potential to be improved is the soundscape of the critical care setting, as the current sound producing events (SPEs) that occur in such settings create a cacophony [21]. Furthermore, overall sound level in the ICU is evidently unhealthy and even hazardous for the patients through the sound-induced complications clinicians have. For example, clinicians suffer from alarm fatigue (i.e., desensitization to medical alarms) due to the constant exposure to the excessive number of alarms, which at times compromises the safety of the patient as some alarms might go unnoticed $[22,23]$. The negative effect of ICU noise on patients' sleep experience is supported by both subjective (sleep assessments with patients and nurses in the critical care setting) and objective evidence (sound measurements). Although the World Health Organization's (WHO) Guidelines for Community Noise advises that the sound level in critical care settings should not exceed $35 \mathrm{dBA}$ on average with peaks of $45 \mathrm{dBA}$, hospitals can rarely live up to these guidelines [24]. An investigation to the ICU sound levels in reference to the WHO guidelines [25] showed that average sound levels in the ICU were constantly exceeded, with average values between 52 and $59 \mathrm{dBA}$ in 
ICU patient rooms half of the time. Moreover, peaks above $85 \mathrm{dBA}$ during the day were no exception amongst the investigated ICUs, even occurring up to 16 times per hour overnight. These measurements are also in line with the results of an ongoing study at Erasmus Medical Centre Rotterdam with one exception that weekends seem to be quieter than weekdays (see Figure 1). According to these studies, sounds levels above $45 \mathrm{dBA}$ seem to remain the norm rather than the exception and given the situation of critically ill patients and their susceptibility for excessive environment noise, an acoustically hostile environment is created.

However, Figure 1 and measurements of sound quality solely focus on $\mathrm{dB}$ (with lack of spectral and temporal content, impulsiveness, and duration) and this provides little information on how hostile the acoustical environment is and how the soundscape and its contributors are actually perceived (loud, noisy, and calm) and attributed meaning to. Especially, the meaning the sound carries (e.g., alarms signal a health threatening events or voices of family members represent moral support) plays a substantial role in the auditory experience of listeners and therefore should not be neglected.

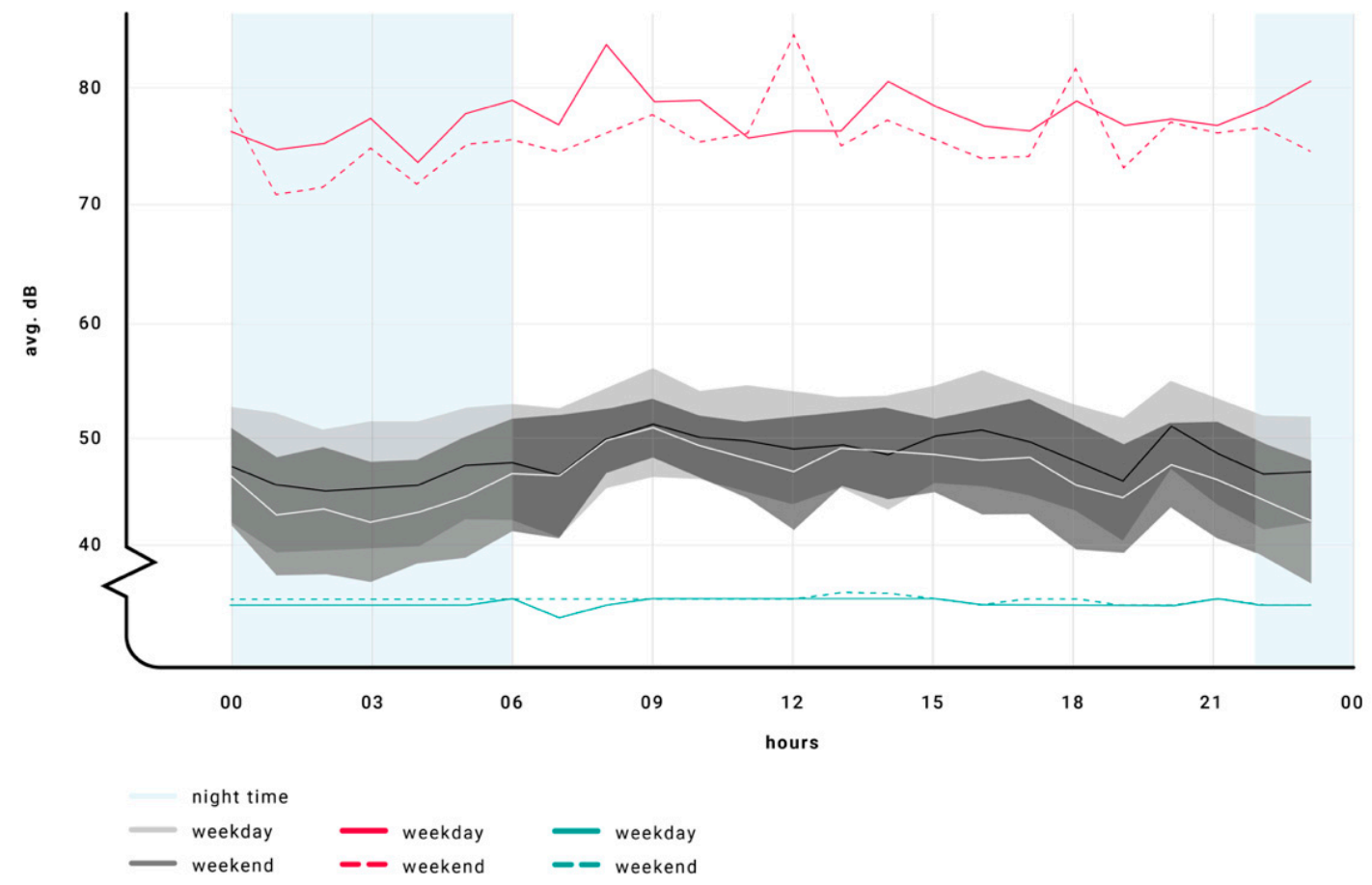

Figure 1. Average sound recordings over the week in an IC patient room at the Erasmus Medical Centre, Rotterdam. The Sound Pressure Level was measured each second over the course of $24 \mathrm{~h}$ and then averaged for weekdays and weekends. The data illustrate that the WHO guidelines are regularly exceeded over the course of the day. Note that these data were measured in the previous IC department of Erasmus MC. Since mid-2018, the department has moved to a newly build IC department, where management of the soundscape has been given more attention. The data were acquired by the authors through the use of Quietyme Inc. sensors.

The aforementioned studies indicate that ICUs are a sonically cluttered, noisy environment. Noise can induce changes in sleep depth and deterioration of sleep quality in the perception of patients and hospital staff $[17,26]$. Although noise is defined as unwanted sound that can affect people psychologically and physiologically [27], this definition does not always apply to the noise experienced in the critical care setting, as certain SPEs (e.g., medical alarms and mechanical ventilator) might be unwanted by patients and even the hospital staff, but of vital importance for the survival of the patient. Consequently, the clinical staff is aware of the high noise levels in the critical care setting, yet they accept it as it seems to be almost unavoidable, even though multiple studies connected the noise levels in the ICU with adverse outcomes for the patient such as delirium [9]. More specifically for the sleep of 
patients in the ICU, a study showed that environmental noise was responsible for $11.5 \%$ of the overall arousals and $17 \%$ of the overall awakenings from sleep [28].

In general, there is a lack of medical sound management and, although awareness about the problem is present amongst medical professionals [29], improvements are scarce. Few hospitals venture to reorganize sound producing events in the night routine and come up with protocols regarding night sounds in critical care (e.g., Night Protocol in the St. Antonius Hospital, Nieuwegein, The Netherlands) [30]. However, sound is present over 24-h span. Daily hectic activities will also have impact on the night routine of the patient, hence the need for (design) interventions that are not limited to the time of day.

A study reviewing the efficacy of non-pharmaceutic sleep-promoting interventions found that some interventions (earplugs and eye masks) may have a beneficial effect on sleep and the incidence of delirium in the population, however the quality of the evidence was low [31]. Furthermore, these design interventions address the symptoms rather than the causes and therefore fail to value the complexity of the problem nor address it in a holistic way. Although the underlying need to provide 24-h care to critically ill patients impacts the prioritization of interventions [29], it is unacceptable that clinicians settle for noisy work conditions and patients and family regard this as normal. Thus, there are cultural issues about sound producing events that need to be tackled from ethnographic and anthropological perspectives as how the society as well can help reduce noise in hospitals. Furthermore, sound producing events should be considered more holistically as part of the critical care soundscape that presents the bigger picture of the sound problem and therefore induces innovative design solutions.

\section{The Soundscape of the Critical Care Environment and Its Acoustical Representation}

Critical care environments are workspaces in which sound is an integral part of the functioning of this environment. The soundscape of critical care environments consists of operational sounds that are typical to the main function of critical care (e.g., medical alarms, machinery sounds of life-support devices; care giving activities) and structural sounds that are typical for larger workspaces but not confined to a certain context (e.g., air-conditioning and elevator doors) (cf. Krueger [32]). Konkani and Oakley [33] further specified the critical care sounds as the following: conversations (among clinical staff, patients and visitors); medical alarms; operational sounds of medical equipment (i.e., cyclic sound of a mechanical ventilator or infusion pumps); caregiving activities (i.e., washing and administering medicines) and incidental sounds (i.e., telephones, pagers, and closing doors).

Critical care soundscape with the aforementioned sound events affects both patients and clinical staff. Figure 2 maps the sound producing events only from the patient perspective to demonstrate the sounds patients are exposed to. Patients' contribution to the soundscape (i.e., bodily sounds such as coughing) is not considered. Clinical staff contribute to the soundscape through caregiving activities such as interacting with alarms and devices, having conversations with each other, and interacting with the building and its components (doors and floors). Sometimes, both nurses and patients are exposed to the peripheral sounds that are emitted by other nurses, care activities or patients. Moreover, the environment such as weather and construction noise outside also influences the soundscape, although they are not directly linked. 


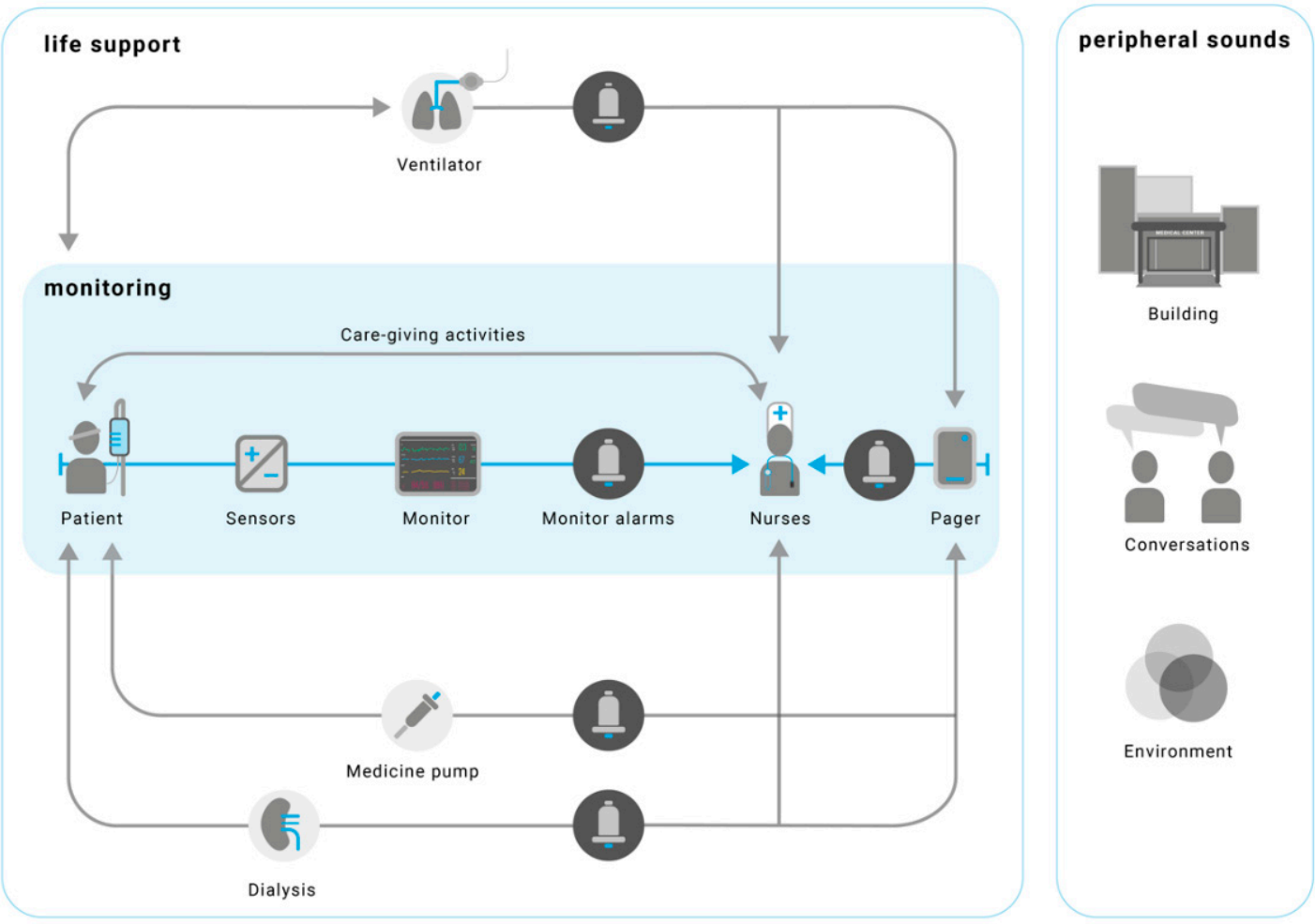

Figure 2. Insight into the distribution of the sound producing events over the critical care sound categories (Figure adopted from Bogers [34]).

In an acoustical evaluation study, Park and colleagues collected $67 \mathrm{~h}$ of audio recordings at the intensive care unit of the Jeroen Bosch Hospital in 's-Hertogenbosch (the Netherlands) [35]. Their study provided insight into the distribution of the sound producing events over the critical care sound categories. With the evident patient involved noise (31\%) excluded, the most prominent contributors to the critical care soundscape were staff related sounds (57\%) and medical alarms (30\%), in which the operational sounds of life supporting devices had a relatively minor contribution (13\%). The staff related sounds were further specified as vocal staff related sounds (38\%) (i.e., verbal and non-verbal sounds of the staff) and sounds related to staff activity (19\%) (i.e., caregiving activities). Although some nuances in the outcomes of the study are in place, it did illustrate that most of the noise created in the critical care soundscape may be attributed to (modifiable) human actions.

Considering this, we argue that the clinical staff is an important factor in the overall acoustical environment of the ICU. Whether it is through SPEs that are beneficial to the patient (caregiving activities) or SPEs that are less in the interest of the patient (non-medical related conversations between members of the clinical staff), noise induced by clinical staff is seen as a significant factor in causing stress in the critically ill patients' perception, as illustrated in previous studies on this topic [27].

Another factor adding significantly to the acoustical environment of the ICU is the medical equipment. Apart from the sounds with a more constant nature (e.g., mechanical ventilation devices) that are hard to prevent, alarms are the biggest polluter in the ICU in terms of sounds. The general consensus amongst studies focusing on alarms in the hospital context is that there are too many pre-emptive (low priority and false) alarms. This is problematic as the abundance of alarms interferes with communication and affects the concentration of the clinical staff, increasing the probability of medical errors and reducing the response rates to the alarms [36].

Underlying mechanisms responsible for causing this situation in the critical care environment are well described in the literature [37]. From a liability perspective, manufacturers choose "a better safe than sorry" approach towards alarm design, resulting in conservative alarms (i.e., sensors are sometimes too sensitive and trigger unactionable alarms). Furthermore, monitoring devices often 
work with a threshold structure (principle of thresholds), which leads to inappropriate use of upper and lower alarms limits, considering that threshold changes are reversible even without intervention of a clinician. Lastly, there is often inappropriate urgency mapping for the alarms, meaning that prioritizing alarms based on the severity of the illness of the patient or the condition of the medical device fails to take place.

Considering the patient population of critical care, it would be safe to assume that exposure to the soundscape of the ICU and the stimuli within hold a great deal of novelty for a significant portion of patients. Critical care sounds are not only acoustically disturbing, they are also difficult to identify as a patient. The entire soundscape as well as the physical context is novel to the patients. Novel sounds are likely to trigger a response in the sleeping brain [38]. This could lead to an arousal in the patient and eventually wake the patient up, subsequently harming the sleep experience of the patient. It is therefore of vital importance to better understand how the critical care soundscape is perceived and what specific sounds play a role in the in the sleep experience of patients. A first step towards creating such an understanding could be by categorizing the sounds in the critical care soundscape to find which SPEs should be excluded in order to improve the acoustical environment for critically ill patients.

\section{Strategy for Designing Sleep-centered Soundscapes in Critical Care}

We acknowledge that sleep is an interplay between many factors (i.e., sleep health), hence our call to consider each sleep-related aspect equally to eventually create a synthesis of approaches. Considering this, we have synthesized our insights into a strategy. This is shown in Figure 3, which indicates five phases. Accordingly, we defined our goal (Phase 1) as improving patient sleep of the critically ill and our context analysis (Phase 2) resulted in understanding the sleep phenomenon in critical care and we collected evidence for how sound producing events might be detrimental to patient sleep with their acoustical properties and looked at the other users of the soundscape (i.e., nurses and other patients) to define their role and interest in the critical care soundscape. Phases 1 and 2 gave rise to three research directions (Phase 3), which are described as the following:

Firstly, we want to emphasize the importance of knowing to what extent specific sound sources in the critical care setting are responsible for deteriorating the sleep experience of critically ill. Gaining insight into this matter is important to better understand what specific SPEs and corresponding sound characteristics (sound pressure level, frequency and intensity) are responsible for triggering arousals in sleeping patients. We argue that this is instrumental to get insight into the current situation and find what sound producing events are relatively seen most disturbing to the patient's sleep experience. It is expected that defining the (sleep) relevant SPEs will eventually lead to a greater understanding of the critical care soundscape.

Secondly, we think that it is essential to analyze if and where to implement data to personalize the hospital experience of patients. Whether these are data gathered inside the hospital or data originating from private healthcare devices (e.g., smartwatches and smartphones), a way should be created to make use of these potentially valuable data.

Finally, the third approach considers the role rather positive sounds can play in improving the sleep experience of patients, thus enabling policymakers and designers to deliberately employ sounds for (patient) well-being, rather than solely focusing on sounds with adverse effects on the patients. In the following sections, we further explain each approach in detail by going through the different knowledge domains (Phase 4) and highlighting examples of (possible) design interventions (Phase 5) that substantiate our view as the first two phases (goal and context analysis) are adequately explained in Sections 1-3. 


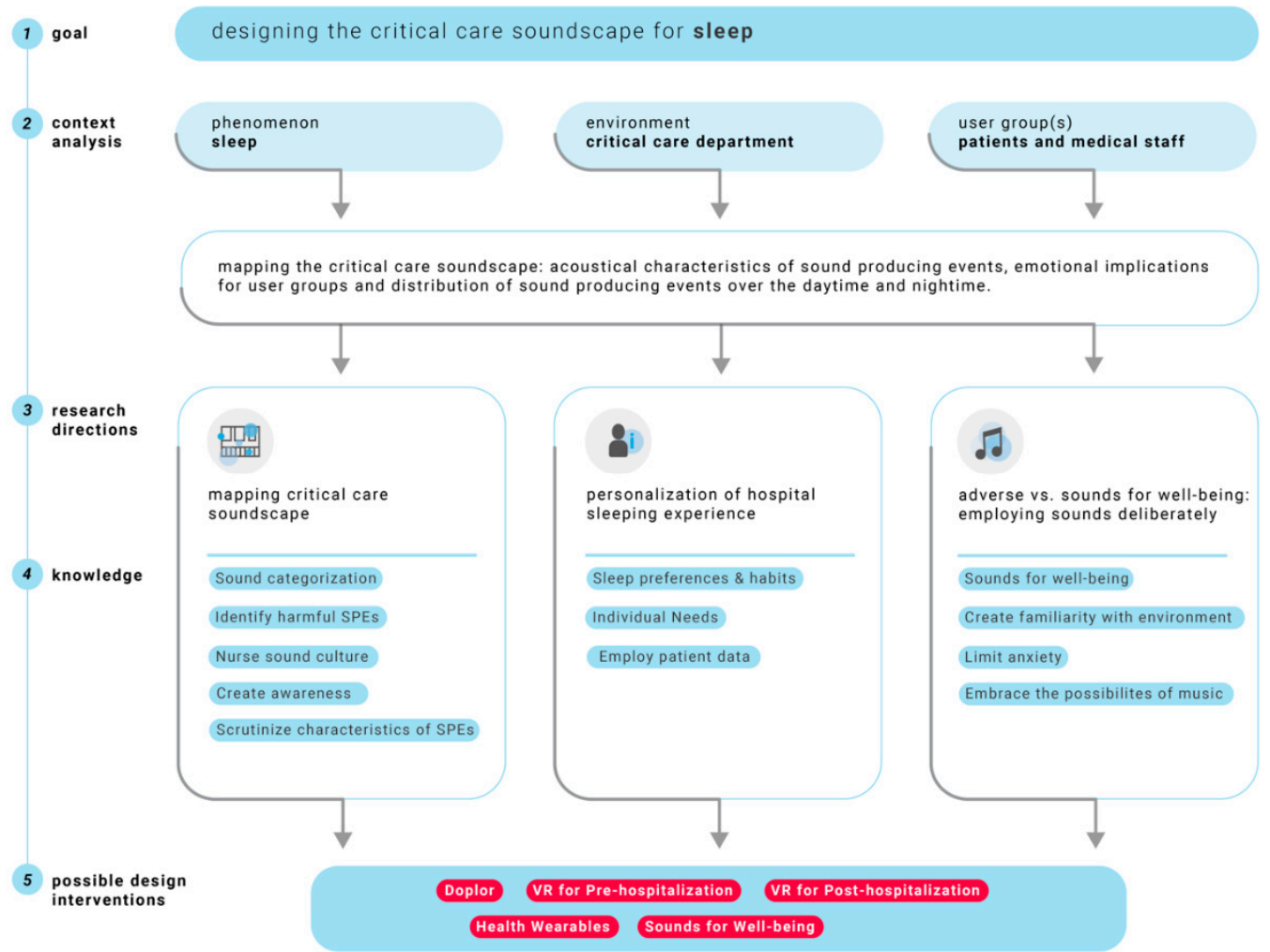

Figure 3. A five-phase strategy to improve the sleep experience in critical care environments.

\subsection{Mapping the Critical Care Soundscape}

\subsubsection{Research Direction}

To further inform approaches that aim to reduce noise in the critical care environment, a better understanding should be created about which SPEs with what acoustic characteristics are most adverse for the patient's sleep experience. Mapping the acoustical environment of the ICU might be a crucial step for creating knowledge on this topic, however equally important would be linking the occurrence of arousals in sleeping patients to specific SPEs. Connecting these outcomes would enable researchers to objectively describe the effect of SPEs on sleep quality, as the number of arousals and arousal depth can be an explanatory measure for assessing this.

It is evident that identifying specific (combinations of) sound characteristics that disturb patients can expand the outlook on the already existing knowledge on this topic. Previous research showed that noise can induce changes in sleep depth and deterioration of sleep quality in the perception of patients and hospital staff $[17,26]$. Sound pressure level is often used as parameter to assess the level of noise in sound measurements. However, sound pressure level alone is not conclusive enough to describe the effect of noise on sleep, impulsiveness, the information contents and frequency compositions of noise have a substantial effect as well. What stands out however is that steep emergence of noise with between baseline and peak causes people to wake up, which consequently means that some impulsive sounds could be masked by constant high background noise, a situation not uncommon to the ICU [39].

It could furthermore reveal which operational sounds are, in relative terms, most harmful for the patients. It might also further substantiate the believe that the sound culture amongst the nurses is a factor negatively impacting the overall soundscape of the critical care environment. When this or other specific SPEs are identified, measures could be taken by hospital management to influence the potential disturbances in the patients sleep experience. It may well be that these disturbances are quite preventable and making the hospital staff more aware of what their actions can cause might suffice. 
Categorization of sounds in terms of events as well as their potential for harm opens up possibilities for design interventions that can change the sound culture in intensive care units.

\subsubsection{Suggestion for Intervention-Doplor}

Making the hospital staff more aware of their influence on the noise in the critical care setting is not necessarily achieved by laying down stricter rules and guidelines regarding sounds. A subtle approach might be equally effective, though less intrusive to the practices and habits of the hospital staff. Doplor is an example of a subtler approach for making hospital staff aware, and while it shows their immediate effect on the critical care soundscape, it is not done in a pedantic way. Doplor is a screen-based device that will measure environment sounds and evaluate if these sounds exceed the set limits. Whether the measured sounds surpass or stay within the limits, Doplor will communicate sound levels through an animated painting displayed on the screen. Doplor conveys this message through metaphors, which should help to communicate in a less enforcing way: a loud environment leads to a hostile visualization on the screen, while a quieter environment eases the visualization to a calmer state (Figure 4) [40].
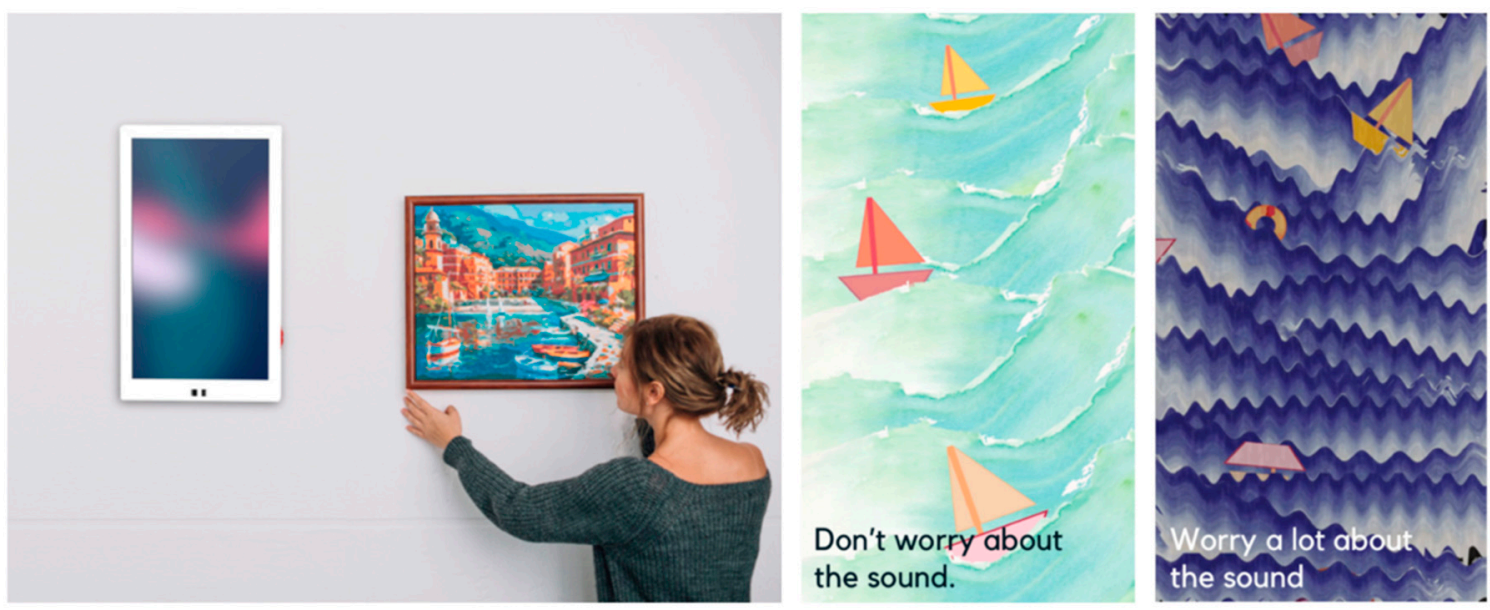

Figure 4. Doplor should function as a wall decoration and subtle tool to improve the noise problem of the critical care soundscape [40].

\subsection{Personalization of Hospital Experience}

\subsubsection{Research Direction}

In the case of noise caused by the clinical staff, solutions should be sought in the hospital protocol that guide daily routines, which should be designed in a way that unwanted SPEs that are not in favor of the patients can be prevented. For example, alarms can be better managed and more systematically coordinated so that one sound, regardless of manufacturer or version of device, signifies one condition. To reduce the amount of non-actionable alarms that can add up to $80-99 \%$ of the alarms occurring in the ICU, alarm management could take place at a centralized place in the hospital, which would subsequently serve as the place where prioritization of alarms can take place [4,22]. Furthermore, centrally managing alarms could facilitate deeper analysis of patient data and allow for better control and perspective on trends in the patient's pathology (e.g., sepsis). Parts of this strategy are already implemented by Emory Healthcare (Georgia, USA), Royal Perth Hospital (Australia) and health-tech company Philips, by means of their eICU, which aims to provide access to medical experts when a bedside intensivist is not available [41]. 


\subsubsection{Suggestion for Intervention-Patient-Specific Alarm Management}

Better alarm management would also require hospitals to reconsider their strategies concerning the use of patient data. Managing data-intensive alarms places patient data right at the heart of the hospital and asks for consent of patients to make use of their data. At the moment, we are at the start of the process towards implementing the use of patient data at all stages of hospitalization, even if it involves data that are generated by patients through personal health devices (e.g., health wearables). This may sound frightening at first considering data privacy and a way has to be found to this within the limits of the General Data Protection Regulation (GDPR). However eventually this will help in the development of more personalized care and that is important because it is expected by society, but equally important, because care practices need to become more efficient to keep them affordable. Future prospects regarding population growth and ageing indicate that the pressure on hospital care and especially critical care will continue to grow. As a consequence, policymakers will be forced to re-evaluate hospital practices for making them more efficient, should the same level of care to be sustained with more limited resources. To achieve this, practices could be more supported by using specific patient profiles based on data gathered inside (through the patient monitoring devices) and outside the hospital (health wearables). These data could range from personal preferences to continuously retrieved data coming from monitoring devices.

\subsubsection{Suggestion for Intervention-Digital Patient Profiles}

When we relate patient profiles to sleep, there is potential to implement sleep preferences of patients before hospitalization, in order to improve their sleep experience during hospitalization. Patients of whom their stay in the hospital is planned before, could monitor their sleeping habits in the weeks leading up to their hospital stay and this information could be used when they are hospitalized. Little adjustments in bedtime or other sleep-related preferences could be the small improvements in the overall interplay of sleep-related factors that make a difference in the sleep experience of patients. Whether legislators or hospitals should take the lead in the process towards more personalized care is unclear, however that it eventually will happen is uncontested.

\subsection{Adverse Sounds vs. Sounds for Wellbeing: Changing the Outlook on the Use of Sounds}

\subsubsection{Research Direction}

Another way of addressing the noise issue in the critical care environment could be to embrace the sounds rather than treat them as something that needs to be avoided. Whether we like it or not sounds will always have their part in critical care settings as vital operations depend on sounds to inform and alert caregivers. Considering ways of employing the soundscape of the critical care environment in a way that it is supportive and even revitalizing, rather than adverse in the patient's perception may hold the potential to make a difference in this issue. Reducing the novelty of the critical care environment could be one of the possibilities for limiting the effect on the patient's sleep experience. This could be done by making patients, of whom the pathology —or treatment path-is foreseeable, acquainted with the critical care environment before admission. In maternity care, it is already common practice that pregnant women have the possibility to attend a tour around the maternity healthcare setting [42]. Caregivers can then answer the questions that the future parents might have and provide information about what they could expect when giving birth. In this way, the novelty of the situation may be reduced and the focus can be on giving birth, rather than being anxious about the unfamiliar environment.

\subsubsection{Suggestion for Intervention-Virtual Reality for (Post) Critical Care Patients}

In the critical care setting, a similar approach that makes use of virtual reality (VR) is getting tested to see whether it can have a positive impact [43]. This approach mainly focuses on the adverse effects that patients have after they have left the intensive care. This condition, of which the effects can 
be physiological and psychological, is better known as post IC syndrome (PICS) and it is estimated that $30-60 \%$ of former critically ill patients are suffering from this. The aim of this approach is to let patients relive the experience they have had in the ICU, but with a better explanation of what was happening with them. It is impossible to give the former patients an actual tour around the IC facilities, hence the choice for letting them relive their IC experience through the VR environment. Similar to cognitive behavioral therapy (CBT) and eye movement desensitization and reprocessing (EMDR), memories are altered and reorganized in a way that they are stored differently because new associations are made, which could allow former patients to have more control over uncontrolled flashbacks. Recreating the situations that critically ill patients endured in the intensive care is expected to have a positive effect.

Depending on the outcomes of the study, this approach may also be applied in other stages of the critical care experience of the patient. Foreseen is that letting patients experience the critical care environment can also be applied pre-IC admission, especially for patients that will have a relatively predictable disease progression. We would like to propose that the critical care soundscape should play an instrumental role if and when this becomes common practice. In this way, the novelty of the critical care soundscape can be reduced, subsequently lowering the effect that novel sounds have on the sleep experience of the patient. It would further create space to think about sounds that can have a positive effect on critically ill patients and use the findings to deliberately design a soundscape that is conducive to the patient.

\subsubsection{Suggestion for Intervention-Music as Opportunity for Critical Care}

Previous (non-clinical and clinical) research showed that the use of music as tool for the (self)-regulation of emotions can be effective [44,45]. A study amongst patients that received acute ventilatory support to prevent respiratory failure showed that patient directed music (PDM) reduced patient anxiety when compared to normal care [46]. The effect of noise cancelling headphones (NCH) on the anxiety levels of patients was considered as well. Comparing PDM and NCH showed that $\mathrm{NCH}$ had a greater effect on reducing anxiety in patients than PDM. The same applied to sedation intensity, patients with $\mathrm{NCH}$ required a lower sedation intensity compared to patients exposed to PDM, with patients receiving normal care requiring the highest sedation intensity. Furthermore, it was demonstrated that the sedation frequency of patients exposed to PDM was lower when compared to $\mathrm{NCH}$ and normal care.

In other areas of critical care, specifically the neonatal intensive care area (NICU), initiatives surrounding the use of music are in further developed state. The National Institute for Infant \& Child Medical Music Therapy, a collaboration between Florida State University and Tallahassee Memorial Healthcare, has been making progress in the field of music therapy. Since 2005, they have supported research and evidence-based clinical practice, and they are currently providing professional training in the efficacy of music therapy for enhancing medical treatment of infants. Previous studies on the effect of music therapy in NICU settings revealed that it may help to reduce respiratory rate and maternal anxiety; evidence about the physiological or behavioral outcomes in the short and long run remains much needed [47].

It seems that this is in line with the consensus among studies regarding music therapy and, despite several studies with promising results to the potential of music in critical care environments, evidence for the effectiveness of music on critically ill patients based on studies with a larger population is absent [46]. More research is therefore needed to be able to establish the short- and long-term effects of music therapy in critical care settings, with the eventual aim to guide future design interventions regarding music therapy.

\section{Conclusions}

In this paper, we propose a comprehensive strategy to improve the patient sleep experience through a holistic approach such as soundscape design. We studied the sleep phenomenon in relation to critical care sounds with the intention to inform hospital management, medical device producers 
and policy makers regarding the complexity of the issue and possible modes of design interventions. We showed that improving patient sleep not only depends on patient related internal factors but also the overall sound quality of the critical care settings and that sound producing events is a main threat for patient sleep as well as wellbeing. We argued that critical care sounds are a culmination of several caregiving activities dependent mainly on the utilization of medical devices, their alarms and interactions among clinicians and between clinicians and patients. We propose innovative design interventions that range from using virtual reality to readjust the auditory memories of former patients of critical care to turning acoustic data into artful warnings for clinicians to quickly assess and adjust the auditory quality of the care settings. We also suggest improved alarm management systems that are catered for individual patient profiles and needs and how adding positive sounds such as music can help change the outlook of critical care environments. Overall, the approaches in our strategy to treat sound producing events as soundscapes, to consider multiple users, and to rethink the major sound-relevant activities around patient care as opportunities for design have the potential to result in patient-centered innovative solutions.

The soundscape design approach presented in this paper is a first step to understanding complex soundscapes with scientific foundations relating to user needs and how the scientific insights can provide design solutions to complex environments. We showed that soundscapes are multi-dimensional entities and their design requires a systematic approach that considers multiple sound producing events as one overlapping auditory input for perception, multiple actors that contribute to the soundscape by engaging in certain sound producing activities and multiple users that have different needs and concerns with sounds. Thus, understanding the sound problem will require more elaborate research into the context of users experience of a certain phenomenon.

We acknowledge that designing for soundscapes also requires a deep knowledge in how perception takes place for the critically ill as well as the nurses on duty and emotionally vulnerable family, as each individual stakeholder in the critical care context (clinical staff, patients and visitors) can experience sounds in a different way. We have reflected on deliberately adding sounds to the critical care soundscape that can have positive effects on anxiety and stress in critically ill patients and we think this could be a valuable addition. However, caution is advised as there is a thin line between potentially harmful and potentially beneficial sounds. We suggest that the ideas generated through this strategy should always be prototyped and tested in controlled conditions.

We demonstrated how this strategy opens up the need for domain specific knowledge that could be used as input for innovative design. Thus, the proposed research directions could be further pursued to discover their scientific potential to inform medical practices and product development. Similarly, some of the design interventions are yet to be discussed further and some are at the stage of clinical trials (e.g., Doplor and virtual reality for critical care). Others, such as musical intervention, require radical change in critical care. In all proposed interventions, we aspired to change how critical care soundscape could be better experienced and solutions for that can be diverse.

This paper should function as a strategic document that sparks discussion between the stakeholders (hospital management, manufacturers of medical devices, and policy makers) involved in the critical care environment and designers aiming to create meaningful interventions. We believe the five-phase strategy presented in Figure 3 could be used for resolving other patient needs in critical care in relation to soundscapes and the involved stakeholders could generate innovative and patient-centered design solutions following this structure.

Author Contributions: Conceptualization, D.B. and E.Ö.; methodology D.B. and E.Ö; formal analysis, D.B. and E.Ö.; investigation, D.B.; resources, E.Ö.; writing—original draft preparation, D.B.; writing—review and editing, D.B. and E.Ö.; visualization, D.B.; supervision, E.Ö.; project administration, E.Ö.; funding acquisition, E.Ö.

Funding: This research entitled "The Silent ICU" was funded by Delft Health Initiative (TU Delft) with the project code Q50266.

Conflicts of Interest: The authors declare no conflict of interest. 


\section{References}

1. Jensen, D.P.; Herr, K.A. Sleeplessness. Nurs. Clin. N. Am. 1993, 28, 385-405.

2. Kristensen, M.S.; Edworthy, J.; Özcan Vieira, E.; Denham, S. Alarm fatigue in the perception of medical soundscapes. In Proceedings of the European Congress and Exposition on Noise Control Engineering, Madrid, Spain, 16-19 June 2019.

3. Darbyshire, J.L. Excessive noise in intensive care units. BMJ 2016, 353, i1956. [CrossRef]

4. Özcan, E.; Birdja, D.; Simonse, L.; Struijs, A. Alarm in the ICU! Envisioning patient monitoring and alarm management in future intensive care units. In Service Design and Service Thinking in Healthcare and Hospital Management; Springer: Berlin, Germany, 2019; pp. 421-446.

5. McKenna, H.; van der Horst, G.T.; Reiss, I.; Martin, D. Clinical chronobiology: A timely consideration in critical care medicine. Crit. Care 2018, 22, 124. [CrossRef] [PubMed]

6. Fox, M.R. The importance of sleep. Nurs. Stand. 1999, 13, 44. [CrossRef]

7. Özcan, E.; Birdja, D.; Edworthy, J.R. A Holistic and Collaborative Approach to Audible Alarm Design. Biomed. Instrum. Technol. 2018, 52, 422-432. [CrossRef]

8. Kondo, Y.; Fuke, R.; Hifumi, T.; Hatakeyama, J.; Takei, T.; Yamakawa, K.; Nishida, O. Early rehabilitation for the prevention of postintensive care syndrome in critically ill patients: A study protocol for a systematic review and meta-analysis. BMJ Open 2017, 7, e013828. [CrossRef] [PubMed]

9. Kamdar, B.B.; Needham, D.M.; Collop, N.A. Sleep deprivation in critical illness: Its role in physical and psychological recovery. J. Intensive Care Med. 2012, 27, 97-111. [CrossRef] [PubMed]

10. ISO 12913-1. Acoustics-soundscape-part 1: Definition and conceptual framework. In Technical Report, International Organization for Standardization; ISO: Geneva, Switzerland, 2014.

11. Adam, K. Sleep as a restorative process and a theory to explain why. In Progress in Brain Research; Elsevier: Amsterdam, The Netherlands, 1980; Chapter 53; pp. 289-305.

12. Roth, T. Daytime sleepiness and alertness. Princ. Pract. Sleep Med. 1989, 48, 14-23.

13. Dahl, R.E.; Lewin, D.S. Pathways to adolescent health sleep regulation and behavior. J. Adolesc. Health 2002, 31, 175-184. [CrossRef]

14. Tembo, A.C.; Parker, V.; Higgins, I. The experience of sleep deprivation in intensive care patients: Findings from a larger hermeneutic phenomenological study. Intensive Crit. Care Nurs. 2013, 29, 310-316. [CrossRef]

15. Pisani, M.A.; Friese, R.S.; Gehlbach, B.K.; Schwab, R.J.; Weinhouse, G.L.; Jones, S.F. Sleep in the intensive care unit. Am. J. Respir. Crit. Care Med. 2015, 191, 731-738. [CrossRef]

16. Friese, R.S.; Diaz-Arrastia, R.; McBride, D.; Frankel, H.; Gentilello, L.M. Quantity and quality of sleep in the surgical intensive care unit: Are our patients sleeping? J. Trauma Acute Care Surg. 2007, 63, 1210-1214. [CrossRef]

17. Parthasarathy, S.; Tobin, M.J. Sleep in the intensive care unit. In Applied Physiology in Intensive Care Medicine; Springer: Berlin, Germany, 2009; pp. 191-200.

18. Elliott, R.; McKinley, S.; Cistulli, P.; Fien, M. Characterisation of sleep in intensive care using, 24-hour polysomnography: An observational study. Crit. Care 2013, 17, R46. [CrossRef]

19. Knauert, M.P.; Yaggi, H.K.; Redeker, N.S.; Murphy, T.E.; Araujo, K.L.; Pisani, M.A. Feasibility study of unattended polysomnography in medical intensive care unit patients. Heart Lung J. Acute Crit. Care 2014, 43, 445-452. [CrossRef]

20. Buysse, D.J. Sleep health: Can we define it? Does it matter? Sleep 2014, 37, 9-17. [CrossRef]

21. Tainter, C.R.; Levine, A.R.; Quraishi, S.A.; Butterly, A.D.; Stahl, D.L.; Eikermann, M.; Lee, J. Noise levels in surgical ICUs are consistently above recommended standards. Criti. Care Med. 2016, 44, 147-152. [CrossRef]

22. Cvach, M. Monitor alarm fatigue: An integrative review. Biomed. Instrum. Technol. 2012, 46, $268-277$. [CrossRef]

23. Kristensen, M.S.; Edworthy, J.; Özcan, E. Alarm fatigue in the ward: An acoustical problem? Sound Eff. An Interdiscip. J. Sound Sound Exp. 2016, 6, 88-104. [CrossRef]

24. Berglund, B.; Lindvall, T.; Schwela, D.H. Guidelines for Community Noise Geneva; World Health Organization: Geneva, Switzerland, 2009.

25. Darbyshire, J.L.; Young, J.D. An investigation of sound levels on intensive care units with reference to the WHO guidelines. Crit. Care 2013, 17, R187. [CrossRef] 
26. Pugh, R.J.; Jones, C.; Griffiths, R.D. The impact of noise in the intensive care unit. In Intensive Care Medicine; Springer: New York, NY, USA, 2007; pp. 942-949.

27. Xie, H.; Kang, J.; Mills, G.H. Clinical review: The impact of noise on patients' sleep and the effectiveness of noise reduction strategies in intensive care units. Crit. Care 2009, 13, 208. [CrossRef]

28. Freedman, N.S.; Gazendam, J.; Levan, L.; Pack, A.I.; Schwab, R.J. Abnormal sleep/wake cycles and the effect of environmental noise on sleep disruption in the intensive care unit. Am. J. Respir. Crit. Care Med. 2001, 163, 451-457. [CrossRef] [PubMed]

29. Eliassen, K.M.; Hopstock, L.A. Sleep promotion in the intensive care unit-a survey of nurses' interventions. Intensive Crit. Care Nurs. 2011, 27, 138-142. [CrossRef]

30. Van de Pol, I.; Van Iterson, M.; Maaskant, J. Effect of nocturnal sound reduction on the incidence of delirium in intensive care unit patients: An interrupted time series analysis. Intensive Crit. Care Nurs. 2017, 41, 18-25. [CrossRef] [PubMed]

31. Hu, R.F.; Jiang, X.Y.; Chen, J.; Zeng, Z.; Chen, X.Y.; Li, Y.; Evans, D.J. Non-pharmacological interventions for sleep promotion in the intensive care unit. Cochrane Database Syst. Rev. 2015, 10. [CrossRef]

32. Krueger, C.; Schue, S.; Parker, L. Neonatal intensive care unit sound levels before and after structural reconstruction. MCN Am. J. Matern. Child Nurs. 2007, 32, 358-362. [CrossRef]

33. Konkani, A.; Oakley, B. Noise in hospital intensive care units-a critical review of a critical topic. J. Crit. Care 2012, 27, 522.e1-522.e9. [CrossRef]

34. Bogers, K. Care Tunes: Music as a Nurses' Monitoring Tool. Master's Thesis, Industrial Design Engineering; Delft University of Technology: Delft, The Netherlands, 2018.

35. Park, M.; Kohlrausch, A.; de Bruijn, W.; de Jager, P.; Simons, K. Analysis of the soundscape in an intensive care unit based on the annotation of an audio recording. J. Acoust. Soc. Am. 2014, 135, 1875-1886. [CrossRef]

36. Edworthy, J.; Hellier, E. Fewer but better auditory alarms will improve patient safety. BMJ Qual. Saf. 2005, 14, 212-215. [CrossRef] [PubMed]

37. Konkani, A.; Oakley, B.; Bauld, T.J. Reducing hospital noise: A review of medical device alarm management. Biomed. Instrum. Technol. 2012, 46, 478-487. [CrossRef] [PubMed]

38. Atienza, M.; Cantero, J.L.; Escera, C. Auditory information processing during human sleep as revealed by event-related brain potentials. Clin. Neurophysiol. 2001, 112, 2031-2045. [CrossRef]

39. Salandin, A.; Arnold, J.; Kornadt, O. Noise in an intensive care unit. J. Acoust. Soc. Am. 2011, 130, 3754-3760. [CrossRef] [PubMed]

40. Redert, R. Doplor: Artful Warnings towards a More Silent Intensive Care. Master's Thesis, Industrial Design Engineering; Delft University of Technology: Delft, The Netherlands, 2018.

41. Christenbury, J. Emory Cares for ICU Patients Remotely, Turning 'Night into Day' from Australia. Available online: https://news.emory.edu/stories/2018/05/buchman-hiddleson_eicu_perth_australia/index. html (accessed on 17 May 2019).

42. Medisch Spectrum Twente. Rondleidingen voor Zwangeren. Available online: https://www.mst.nl/p/Centra/ vkc/bevallen-in-het-vkc/rondleidingen-voor-zwangeren/ (accessed on 17 May 2019).

43. Van Genderen, M.E.; Vlake, J.H. Virtual healthcare; use of virtual, augmented and mixed reality. Ned. Tijdschr. Voor Geneeskd 2019, 163, D3229.

44. Van der Heijden, M.J.; Jeekel, J.; Rode, H.; Cox, S.; van Rosmalen, J.; Hunink, M.G.; van Dijk, M. can live music therapy reduce distress and pain in children with burns after wound care procedures? A randomised controlled trial. Music Med. 2018, 44, 87.

45. Uhlig, S.; Jaschke, A.; Scherder, E. Effects of music on emotion regulation: A systematic literature review. In Proceedings of the 3rd International Conference on Music \& Emotion, Jyväskylä, Finland, 11-15 June 2013.

46. DellaVolpe, J.D.; Huang, D.T. Is there a role for music in the ICU? Crit. Care 2015, 19, 17. [CrossRef]

47. Bieleninik, Ł.; Ghetti, C.; Gold, C. Music therapy for preterm infants and their parents: A meta-analysis. Pediatrics 2016, 138, e20160971. [CrossRef] [PubMed]

(C) 2019 by the authors. Licensee MDPI, Basel, Switzerland. This article is an open access article distributed under the terms and conditions of the Creative Commons Attribution (CC BY) license (http://creativecommons.org/licenses/by/4.0/). 